E.L.U.A., 5, 1988-1989, pp. 207-216

\title{
DOS CASOS DE INTERLENGUAJE EN REGISTRO ESCRITO EN SANTO DOMINGO (SIGLO XIX). LOS DOCUMENTOS EN ESPAÑOL DE TOUSSAINT L'OUVERTURE Y DEL PRESIDENTE BOYER
}

\author{
GERMAN DE GRANDA \\ (Universidad de Valladolid)
}

El concepto de interlenguaje ha sido considerado como uno de los instrumentos básicos de investigación en la lingüística aplicada al aprendizaje de idiomas ${ }^{1}$ incluso con anterioridad al ya clásico artículo de L. Selinker ${ }^{2}$ en el cual, además de poner en circulación definitivamente esta denominación técnica, se perfila cuidadosamente su contenido teórico y se precisan, exigentemente, sus modalidades de aplicación a diferentes ámbitos de estudio.No es, por lo tanto, sorprendente, si tenemos en cuenta de modo adecuado la cada vez más estrecha interrelación entre los diversos enfoques metodológicos que se han venido proponiendo recientemente en cada una de las dimensiones teóricas de la ciencia lingüística, que tal concepto haya sido también utilizado con excelentes resultados, como punto de partida o como concepto fundamental de referencia, en estudios referidos tanto a diversos aspectos de la problemática del contacto lingüístico sincrónico ${ }^{3}$ como a enfoques, de índole diacrónica, conexos con el cambio lingüístico acontecido en el eje temporal ${ }^{4}$. Es evidente que las aplicaciones de la noción de interlenguaje a campos de investigación tan diferentes a aquél para el que fue concebida originariamente ha requerido, sin duda, algunas modificaciones en su delineamiento conceptual ${ }^{5}$ pero conservando siempre, en el renovado perfil teórico forjado para su adecuación instrumental a nuevos

${ }^{1}$ Crr., por ejemplo, S. P. Corder "The significance of learner's crrors", en Intemational Review of Applied Linguistics, 5, 1967, págs. 161-171.

2 "Intcrlanguagc", un International Review of Applied Linguistics, 10, 1972, págs. 209-231.

${ }^{3}$ Véanse, entre otros, R. Appel y P. Muysken Language Contact and Bilingualism, Londres, 1987, y P. Trudgill Dialects in Contact, ()xford, 1986.

${ }^{4}$ Cfr. Sarah Grey Thomason y Terrence Kaulman Language Contact, Creolization, and Genetic Linguistics, Berkeley, 1988.

${ }^{5}$ Véanse las acertadas apreciaciones sobre este tema que exponen S. G. Thomason y T. Kaufman en la obra citada en nota 4 , págs. 145-146. 
ámbitos de uso, su fundamentación básica de carácter gnoseológico.

Quizá podríamos definir, en sus líneas esenciales, el concepto de interlenguaje aplicable a la totalidad de las facetas de estudio hasta aquí mencionadas considerándolo como la noción que abarca las diferentes variedades o estadios de desarrollo que se dan en los hablantes de una lengua fuente $\left(\mathrm{L}_{1}\right)$ antes de llegar al completo dominio de otro código de comunicación $\left(\mathrm{L}_{2}\right)$ considerado como lengua objetivo.

En el transcurso de las investigaciones que se han venido realizando, en los últimos decenios, sobre los factores causales determinantes de los rasgos caracterizadores de los diversos hechos linguísticos relacionables con la noción teórica de interlenguaje se han propuesto dos posturas metodológicas, claramente divergentes en su formulación originaria aunque, como veremos, susceptibles de ser consideradas complementarias la una de la otra en un planteamiento, más abarcador, de índole globalizadora.

La primera de ellas, denominada generalmente hipótesis del análisis contrastivo (C.A.H.), atribuye la producción de las caraterísticas tipológicas del interlenguaje a la interferencia sobre los esquemas gramaticales de la lengua objetivo $\left(\mathrm{L}_{2}\right)$ de los propios de la lengua fuente $\left(\mathrm{L}_{1}\right)$ adoptando, en esta forma de actitud, una postura teórica abundantemente respaldada no sólo por la bibliografía lingüística anterior ${ }^{6}$ sino también por resultados de trabajos experimentales recientes ${ }^{7}$. Por otra parte los partidarios de la denominada hipótesis de la identidad (I.H.), ampliamente preponderantes en la mayor parte de la década de 1970, propugnan el carácter irrelevante de los procesos de interferencia en la génesis de los fenómenos de interlenguaje y atribuyen éstos con exclusividad (en la versión más radical de esta línea de teorización) al resultado de aplicar a los esquemas gramaticales de la lengua objetivo $\left(L_{2}\right)$ estrategias de aprendizaje homólogas a las desarrolladas en la adquisición de la lengua fuente $\left(\mathbf{L}_{\mathbf{8}}\right)$ y, en especial, las caracterizables como procesos de generalización y de simplificación ${ }^{8}$.

Desde los años finales de la década de 1970 , y como consecuencia de los resultados alcanzados en varios estudios de campo atingentes al tema ${ }^{9}$, recobra fuerza explicativa como factor propulsor de los rasgos caracterizadores del interlenguaje el fenómeno de la interferencia de $\mathrm{L}_{1}$ sobre $\mathrm{L}_{2}$. Como consecuencia de ello, y de los serios intentos realizados para hacer compatibles las formulaciones teóricas de la nuevamente valorada tesis de

${ }^{6}$ Cfr. entre otras obras, la clásica de U. Weinreich Languages in Contact: Findings and Problems, La Haya, 1953. También R. Lado Linguistics across Culuures, Ann Arbor, 1957.

7 Véanse, entre otros, L. Duskova "On sources of crrors in forcign language learning", en International Review of Applied Linguistics, 7, 1969, págs. 11-33. Desde una perspectiva diferente cfr. P. Muysken "Halfway between Quechua and Spanish: the case for relexification", en Highlield y A. Valdman (Eds.) Historicity and Variation in Creole Studies, Ann Arbor, 1981, págs. 52-78.

${ }^{8}$ Cfr. H. C. Dulay y M. K. Burt "Errors and stralegies in child second language acquisition", en TESOL Quartery, 8, 1974, págs. 129-136 y "Natural sequences in child second language acquisition", en Language Learning, 24, 1974, págs. 37-53; J. Schachter "An error in error analysis" en Language Learning, 24, 1974, págs. 205-214 y K. Hakuta "A case study of a Japanese child learning English as a second language", en Language Leaming, 26, 1976, págs. 321-351. Como formulación reciente de este punto de vista véase H. Dulay, M. Burt y S. Krasnen Language Two, Nueva York-Cxford, 1982.

9 Cfr., entre otros, H. Zobl "The formal and developmental selectivity of $\mathrm{L}_{1}$ influences on $\mathrm{L}_{2}$ acquisition", en Language Learning, 10, 1980, págs. 43-57 y C. Pfaff " $\left(\right.$ ) input and residual $\mathrm{L}_{1}$ transfer effects in Turkish and Greck children's (jerman", en R. W. Andersen (ed.) Second Languages: A Cross-Linguistic Perspective, Rowlwy, 1984, págs. 271-298. 
la C.A.H. con las de la I.H., se llega a una apreciación, convergente e integradora, de ambas posiciones metodológicas en la que se destaca, ante todo, la importancia de diferenciar no sólo cronológica sino, y sobre todo, metodológicamente los diferentes estadios de aprendizaje abarcados en la consideración general de interlenguaje. Los estadios iniciales del proceso de adquisición de $\mathrm{L}_{2}$ serían primordialmente configurados mediante estrategias, de percepción y realización, similares a las utilizadas en la formación de $\mathbf{L}_{1} \mathrm{y}$, especialmente, a través de la producción de estructuras gramaticales de $\mathrm{L}_{2}$ caracterizables por fenómenos de generalización y simplificación mientras que, por el contrario, los estadios posteriores de interlenguaje, más cercanos a la reproducción adecuada de los sistemas gramaticales de la lengua objetivo, se caracterizarían (principal aunque no exclusivamente) por el desarrollo de rasgos lingüísticos derivados de la interferencia de las estructuras de la lengua fuente $\left(\mathrm{L}_{1}\right)$ sobre las propias de la lengua objetivo $\left(\mathrm{L}_{2}\right)$, situación que se mantendría, si bien en realización progresivamente decreciente, hasta llegar al estadio, final, de adquisición total del código comunicativo que aquí hemos denominado $L_{2}$ (lengua objetivo del proceso de aprendizaje considerado) ${ }^{10}$.

Los materiales que a continuación presentaré quizá puedan facilitar algunos datos en apoyo de esta última alternativa teórica en el análisis de los factores determinadores de los rasgos lingüísticos que se integran bajo la denominación general de interlenguaje ya que en ellos, como se verá en las páginas siguientes, aparecen abundantes fenómenos derivados, sin duda, de procesos de interferencia de la lengua fuente $\left(\mathrm{L}_{1}\right)$ sobre la lengua objetivo $\left(\mathrm{L}_{2}\right)$ y, por el contrario, no se confirma la presencia de los rasgos propios (de simplificación y/o generalización estructural), que deberían producirse según los presupuestos e inferencias teóricas de la llamada hipótesis de la indentidad. Lo que, teniendo en cuenta debidamente el hecho de que los materiales en cuestión representan, de modo claro, estadios no iniciales de interlenguaje, es coherente con los puntos de vista teóricos antes mencionados, de carácter globalizante e integrador, de $\mathbf{H}$. Wode ${ }^{11}, \mathrm{H}$. Zobl ${ }^{12}$ y otros investigadores actuales del proceso de adquisición lingüística.

Creo, sin embargo, que más importantes que su (evidentemente modesta) aportación a la controversia teórica y metodológica sobre la génesis de los rasgos propios del interlenguaje son otras dos notas caracterizadoras de los materiales aquí considerados. Es la primera de ellas su evidente rareza ya que el mismo carácter intrínseco de los fenómenos de interlenguaje hace que sea muy poco frecuente el que textos de esta índole sean presentados por medio del registro escrito. $Y$, en segundo (pero no menos importante) lugar, que los documentos en cuestión facilitan valiosos testimonios históricos sobre un secular proceso de contacto lingüístico (el que ha tenido lugar entre el español y el francés o/y criollo haitiano en la Isla Española) respecto a cuyo interés e importancia, normalmente desconocidos o infravalorados por los estudiosos no relacionados

\footnotetext{
${ }^{10}$ Véanse H. Wodc Leaming a Second Language. 1. An Integrated View of Language Acquisition, Tübingen, 1981; H. Zobl "A direction for contrastive analysis. The comparative study of developmental sequences", en TESOL Quarterly, 16, 1982, págs. 169-183 y B. Le Compagnon "Interference and overgencralisation in second language lcarning. The acquisition of English dative verbs by native speakers of French", en Language Learning, 34, 1984, págs. 30-67. También J. Mcisel et alii "On determining development stages in natural second language acquisition", en Studies in Second Language Acquisition, 3, 1981, págs. 109-125.
}

11 Cfr. ob, cit, en nota 10 .

${ }^{12}$ Cfr. Irabajos cilados en notas 10 y 11 . 
directamente con el área geográfica ${ }^{13}$, ha llamado la atención, muy justamente, en trabajo reciente Irene Pérez Guerra ${ }^{14}$.

Los materiales a que me refiero están constituidos por dos conjuntos documentales, diferenciados tanto por su fecha de redacción como por otras características externas e internas pero relacionados entre sí de modo claro por su tipología lingüística ya que en ambos casos se trata de textos escritos representativos del interlenguaje producido por hablantes de origen haitiano y de lengua francesa que intentan expresarse en español, código lingüístico éste que sólo es manejado de modo claramente inadecuado y parcial por sus redactores.

El primero de estos conjuntos documentales está formado por ocho proclamas y disposiciones oficiales ${ }^{15}$ suscritas por el caudillo haitiano Toussaint L'Ouverture durante su breve permanencia en Santo Domingo (1801-1802) después de haber ocupado con sus tropas negras la parte este de la Isla, antes bajo el dominio español, como consecuencia de la aplicación del nefasto Tratado de Basilea (1795) suscrito entre España y Francia, en el que se preveía la entrega del Santo Domingo español a esta última potencia ${ }^{16}$. El segundo consta de un sólo documento: la proclama, en español, suscrita por el Presidente haitiano Jean Pierre Boyer el 9 de Febrero de $1822{ }^{17}$ en ocasión de la anexión de la zona española de Santo Domingo a la República de Haití ${ }^{18}$.

Recogeré a continuación los rasgos más significativos de los textos en cuestión

${ }^{13}$ Así, por ejemplo, no es ni siquiera mencionado este proceso de contacto lingüístico en el trabajo, por otra parte excelente como panorama de conjunto, de M. B. Fontanella de Weinberg "Contactos lingǘísticos del español americano", en Cuadernos del Sur, (Bahía Blanca, Argentina), 16, 1983, págs. 27-49.

14 "Contactos lingüísticos en la República Dominicana. Su tipología", comunicación presentada al VIII Congreso Internacional de la Asociación de Lingüística y Filología de América Latina (A.L.F.A.L.) que tuvo lugar en Tucumán (Argentina) en 1987. En prensa en las Actas del mencionado Congreso.

15 Estos documentos se encuentran en el Archivo (ieneral de Indias de Sevilla (Estado, 59) y han sido cuidadosamente transcritos en EME EME. Estudios Dominicanos (Santiago de los Cáalleros, República Dominicana), núm. 12, 1974, págs. 146-161. En adelante me referiré a estos textos, en su reproducción mencionada, mediante la abreviatura $\mathrm{L}$ seguida del número de la página citada.

${ }^{16}$ Sobre la personalidad de Toussaint L'Overture véase C..L.R. James, The Black Jacobins, Nueva York, 1963. Acerca del Tratado de Basilca y sus consecucncias en la parte española de Santo Domingo cfr. J. Marino Incháusicgui Documentos para estudio. Marco de la época y problemas del Tratado de Basilea de 1795 en la parte española de Santo Domingo, Bucnos Aires, 1957; Frank Moya Pons Historia colonial de Santo Domingo, Santiago de los Caballeros, 1977 (especialmente capítulos XV y XVI) y Emilio Rodríguez Demorizi Cesión de Santo Domingo a Francia, Ciudad Trujillo, 1955.

${ }^{17}$ Se trata del texio español de la "Proclamation, en français et en espagnol, au peuple à l'ocassion de la réunion de l'Est à la Republique" publicado en M. Linstant Pradine Recueil Ciénéral des Lois et Actes du Gouvernement d'Haiti, III, Paris, 1861), pág. 442. El texto en cuestión ha sido reproducido como apéndice del volumen de Frank Moya Pons La dominación haitiana. 1822-1844, Santiago de los Caballeros, 1978 (págs. 173176). Citaré por esta reproducción, designada mediante la abreviatura $\mathbf{B}$.

${ }^{18}$ Sobre este hecho histórico, comienzo de un período de ocupación del territorio hoy dominicano por Haití que se prolongará hasta 1844 , véase el volumen de F. Moya Pons citado en la nota anterior y cl de E. Rodrígue\% Demorizi mencionado en último lugar en la nota 16. También Jcan Price Mars La República de Haití y la República Dominicana. Diversos aspectos de un problema histórico, geográfico y etnográfico, Puerto Príncipe, 1953. 
haciendo notar previamente que, por haber dedicado ya una monografía ${ }^{19}$ a las características léxicas del material aquí manejado, me limitaré ahora a analizar los datos del mismo que, en aplicación del punto de vista teórico que aquí hemos adoptado, se encuentren exclusivamente en el nivel lingüístico morfosintáctico.

\section{1.- Eliminación de $a$ con objeto directo de persona.}

Es sobradamente conocida la relevancia tipológica que reviste el objeto preposicional del español dentro de las lenguas románicas ${ }^{20}$ y, del mismo modo, han sido estudiados abundantemente su origen ${ }^{21}$, progresiva difusión ${ }^{23}$ y condicionamientos contextuales ${ }^{23}$. En los textos que aquí consideramos la omisión de $a$ ante objeto directo personal es prácticamente completa por lo que no parece admisible atribuir este hecho a la preservación arcaizante de situaciones sintácticas anteriores en el eje diacrónico, de modo diferente a lo que aconteció, casi contemporáneamente, en otras áreas del español americano durante el siglo XVIII si bien, en estos últimos casos, con características considerablemente menos marcadas que en el que nos ocupa ${ }^{24}$. La interferencia del francés parece ser el factor causal exclusivo o, cuando menos, ampliamente predominante en la producción de enunciados como los que siguen: "algunos hombres mal intencionados se dedican a inspirar terrores capaces de excitar los ciudadanos déviles, y fáciles a engañar a emigrarse de la Colonia" 25; "convido igualmente todos los buenos ciudadanos... a volverse" 26;"yo no quiero aspirar a otra Gloria que la de rendir todos los Havitantes de Santo Domingo indistintamente felices y pasibles posehedores de su propiedades" ${ }^{27}$; "yo

19 "Dos episodios en el proceso de interferencia léxica del francés sobre el español dominicano (Toussaint L'Overture, 1801; Boycr, 1822)", en prensá en Homenaje al Prof. Rubio García, Murcia.

20 Cfr. A. Niculescu "Sur l'objet direct prépositionnel dans les langues romanes", en Recueil d'études romanes, Bucarest, 1959, págs. 167-185 y (i. Rohlfs "Autour de l'accusatif prépositionnel dans les langues romancs (concordances ct discordances)", en Revue de Linguistique Romane, 35, 1971, págs. 312-334.

21 Crr. H. Meier "Sobre as origens do acusativo preposicional nas línguas românicas", en Ensaios de Filología Romanica, Lisboa, 1948, págs. 115-164.

22 G. Reichenkron "Das präpositionale Akkusativ-Objckt in ältesten Spanisch", en Romunische Forschunngen, 63, 1951, págs. 342-397; M. A. Martín Zorraquino "A + objeto directo en el Cantar de Mio Cid" en Mélanges offerts a C. Th. Gossen, II, Berna-Lieja, 1976, págs. 555-566 y C. Monedero Carrillo de Albornoz. "El objeto directo preposicional en textos medievales (nombres propios de persona y lítulos de dignidad)", en Boletín de la Real Academia Española, 63, 1983, págs. 241-302.

${ }^{23}$ Cfr. Anna (;. Hatcher "The use of $a$ as a designation of the personal accusative in Spanish", en Modern Language Notes, 57, 1942, págs. 421-429; M. Molho "La question de l'objet en espagnol", en Vox Romanica, 17, 1958, págs. 209-219 y B. Pottier "L'emploi de la préposition a devant l'objet en espagnol", en Bulletin de la Socielé de Linguistique de Paris, 63, 1968, págs. 83-95.

${ }^{24}$ Por ejemplo en Perú. Véase Tomás Bucsa Oliver "Algunos usos preposicionales en textos peruanos del siglo XVII", en Hommage a Bernard Pottier, 1, París, 1988, págs. 115-126 (especialmentc pág. 117).

$25 \mathrm{~L}, 146$.

${ }^{26} \mathrm{~L}, 147$.

27 L, 147. 
me complazco de hacerles oi todos la Justicia que merecen por este motivo" ${ }^{28}$; "El General de Brigada Moisés, se portó con el valor, moderación y brabura, que caracterizan un General francés" 29; "inacesibles a la entrega y a la corrupción y resueltos a despojarse de las pasioncillas que constrinen el espíritu y embilecen los hombres" ${ }^{30}$.

2.- Omisión de artículo tras todos/todas y ante sustantivo.

Calcando la estructura homóloga del francés, se elimina sistemáticamente el artículo que en español se inserta entre todos/todas y el sustantivo que le sigue. Así en casos como "Todos Santos Louverture General en Gefe de los Exércitos de Santo Domingo" 31; "Convido igualmente todos los buenos ciudadanos que han salido por el efecto del pavor a volverse, $y$ traer consigo las personas de todos colores que han salido con éllos" ${ }_{2}$; "El interés que tomo en el suceso de vuestros útiles trabajos me conducirá a redoblar mi vilgilancia, a allanaros todas dificultades" 33 .

3.- Utilización redundante de pronombre personal sujeto.

Se verifica en los documentos que analizamos el empleo sistemático, según el modelo de la sintaxis francesa, de sujeto pronominal expreso ante el verbo en contextos en que tal rasgo no se encuentra justificado por factores como el énfasis, la focalización o la finalidad expresiva claramente marcada. Así en casos como los siguientes: "Yo convido a los Havitantes de la parte antes Española actualmente reunida a la República francesa, a apartar de sus espiritos el terror vano" ${ }^{34}$; "Yo me complazco de hacerles oi toda la Justicia que merecen" 35; "Yo aseguro con todo mi corazón, y en virtud del mando que tengo, que no hai hombre con más firme voluntad y al mismo tiempo más interesado en hacer su Pais feliz que yo" 36; "Ciudadanos vosotros haveis sido Testigos de mi solicitud por la felicidad de mi Pais, por la livertad de mis Hermanos" ${ }^{37}$; "Y vos, ciudadanos de la parte del Este, vos habéis sido desgraciados por largo tiempo" ${ }^{38}$.

\footnotetext{
${ }^{28}$ L, 149.

${ }^{2 y}$ L, 149.

${ }^{30}$ L, 152.

${ }^{31} \mathrm{~L}, 146$.

32 L, 147.

${ }^{33}$ L, 152.

${ }^{34} \mathrm{~L}, 146$.

${ }^{35} \mathrm{~L}, 149$.

${ }^{36} \mathrm{~L}, 160$.

$37 \mathrm{~L}, 151$.

${ }^{38} \mathrm{~B}, 174$.
} 
4.- Empleo de que con valor de 'quienes'.

Sobre el modelo del francés qui se utiliza la forma que con significado equivalente a 'quien/quienes'. Así en el texto que sigue: "Ahora sois vosotros ciudadanos Magistrados que teneis que asegurar a la Colonia su tranquilidad futura" ${ }^{39}$.

5.- Usos interferidos de la preposición en.

Entre los empleos de la preposición en derivados de procesos de interferencia del código de comunicación francés destacan los que siguen. En 'como': "En padre lleno de bondad es el que havía a sus hijos el que les muestra la senda por donde han de lograr la felicidad y la de sus familias y el que desea verlos satisfechos" 40; "Que los antiguos y nuevos avitantes se persuadan que encontrarán siempre en mí un amigo, en un Gefe constantemente dispuesto a ayudarles" ${ }^{41}$. En 'a'. "El derecho de 20 por ciento establecido por el reglamento de 21 Frimario sobre mercancías importadas en la Colonia y las mercancias exportadas de ella, es modificado para los Departtamentos del Yngano y Samaná" ${ }^{42}$.

6.- Usos interferidos de la preposición de.

De con valor partitivo (fr. des): "Vosotros en consecuencia devereis elejir hombres sabios, próvidos, ilustrados... De ciudadanos sin preocupaciones igualmente recomendables por sus Talentos que por su virtudes" ${ }^{43}$.

De con valor final: "con brazos inteligencia y actividad los hombres Lavoriosos tendran certteza de un terreno tan fertil de sacar un premio centuplo de su trabajo y dispendio" 44 .

7.- Usos interferidos de la preposición $a$.

$A$ 'en': "Os anuncio con la mayor satisfacción haver tomado posesión de la parte Española de Santo Domingo a nombre de la República Francesa" ${ }^{45}$.

$A$ 'para': "poner la causa de su prosperidad por medio de leyes útiles a nuestras costumbres, a nuestros estilos, a nuestro clima, a nuestra industria, y del mismo modo propias a adherirnos más todavía más fuertemente a la República Francesa" ${ }^{46}$.

$A$. 'por': "Los Diputtados de la Asamblea Central serán nombrados a pluralidad de

${ }^{39} \mathrm{~L}, 151$.

${ }^{40}$ L, 156-157.

${ }^{41}$ L, 160.

${ }^{42}$ L, 160.

${ }^{43}$ L, 151-152.

${ }^{44} \mathrm{~L}, 159$.

${ }^{45}$ L, 148 .

${ }^{46} \mathrm{~L}, 151$. 
vottos" 47.

8.- Uso interferido de la conjunción $y$.

Me parece extremadamente importante dentro de la línea de investigación adoptada la transferencia, en uno de los documentos que analizamos, del valor pronominal del $y$ francés a la conjunción española y la cual adquiere el contenido semántico de 'en ellos': "Se establecerá en cada Pueblo de la Colonia una compañía de hombres de Armas compuesta de 54 yndividuos y comprendidos tres Oficiales y un Trompetta" ${ }^{48}$.

9.- Que galicado.

Los textos que aqui analizamos facilitan una extensa lista de ejemplos de que galicado entre los que pueden encontrarse un buen número de las modalidades de este fenómeno que han sido clasificadas como tales por los especialistas en esta índole de estudios ${ }^{49}$. Véanse los pasajes siguientes, seleccionados entre otros muchos. "Los Gefes de Brigada Pablo Louverture y Carlos Bilite se han particularmente distinguido en ésta operación tanto por su asiento, discernimiento, y constancia en mantener el soldado en el órden de ovediencia, de Disciplina, y de Subordinación, que de ejecuttar y hacer ejecuttar mis órdenes" so; "En consecuencia de ésto, es que lo expongo al público por ser cierto" 51: "Mi destino era sin duda el instrumento de que debía servirse la Divinidad para hacer triunfar nuestra sagrada causa: solo a su protección es que atribuyo los sucesos que han acompañado mi administración" $\mathbf{5 2}$.

10.- Empleo de sea...sea con significado disyuntivo.

Sobre un modelo francés (soit...soit) se ha forjado en el interlenguaje objeto de nuestro análisis el esquema disyuntivo sea...sea, que sustituye en el mismo a las construcciones castellanas ya...ya, bien...bien, o...o y equivalentes: "Todas las Administraciones sea individual sea colecticiamente, todos los ciudadanos en General son combocados a dar sus Vottos" 53 .

11.- Uso del futuro de indicativo en sustitución del subjuntivo en oraciones de contenido temporal.

El rasgo a que aquí nos referimos, aunque coincide (al igual que ocurre con otros ya mencionados) con posibilidades existentes en la sintaxis castellana medieval y clásica y especialmente en la utilizada en los territorios más orientales del área castellano hablante peninsular, no me parece que pueda ser analizado, dados sus condicionamientos espaciotemporales, sino como un claro fenómeno de interferencia de las estructuras
${ }^{47} \mathrm{~L}, 153$.
${ }^{48} \mathbf{L}, 157$.
49 Véase Rufino J. Cucrvo Apuntaciones críticas sobre el lenguaje bogotano, Bogotá, 1955, págs. 493-516.
So L, 149 .
51 L, 149.
52 B, 175.
${ }^{53} \mathrm{~L}, 153$. 
morfosintácticas del francés. Véanse los fragmentos textuales siguientes: "los proyectos que se propondrán por vosotros en ventaja de esta Colonia serán acogidos con respeto y conocimiento" 54; "Esta Compañía de hombres de Armas, será pagada, vestida y montada, por el Pueblo en cuia Jurisdicción hará el Servicio" ${ }^{55}$.

\section{2.- Construcciones verbales con de.}

Reflejan, evidentemente, fenómenos de interferencia del francés los numerosos casos en que los complementos verbales son precedidos, en los documentos que estudiamos, por de. Téngase en cuenta que los casos aquí reseñados no son incluibles, en cuanto al contenido semántico del verbo, en la categoría de construcciones sintácticas de índole proyectiva, para la cual sería quizá posible sugerir otro tipo de motivación, la de la retención arcaizante ${ }^{56}$. Véanse los ejemplos siguientes: "Yo me complazco de hacerles oi todos la Justicia que merecen con éste motivo" ${ }^{57}$; "En consequencia, se manda y ordena todos los havitantes de la parte Española que posean havitaciones que se dediquen de plantar caña, café, algodón y cacao" 58; "Libre ahora de todos los obstáculos que me han impedido de poner todos mis cuidados a hacer bolver la prosperidad general ayudado por los esfuerzos de los amigos de la Colonia" 59.

13.- Reemplazo sistemático de construcciones pasivas reflejas por formas verbales pasivas.

De acuerdo con la tendencia francesa, especialmente notable en la prosa jurídica, al empleo mayoritario de formas verbales pasivas, en los textos que aquí analizamos prepondera este tipo de construcciones morfosintácticas sobre las de índole pasiva refleja, mucho más utilizadas en la prosa española, incluso en la de contenido legal. Veánse, como ejemplos de ello, los pasajes siguientes: "Esta expresamente mandado a todos los Comandantes Militares de los Departamentos del Ingano y Samaná que hasta que hayan recivido nuevas órdenes de mi parte que hagan aprender y poner presos a toda persona que conduzca animales de un Pueblo a otro" 60; "El derecho de 20 por ciento ... es modificado para los Departamentos del Yngano y Samaná y difinitivamente fijado a 6 por ciento de entrada y 6 por ciento de salida" ${ }^{1}$; "Todas las Administraciones sea individual sea colecticiamente, todos los ciudadanos en General son combocados a dar sus Vottos y a

${ }^{\mathbf{S 4}} \mathrm{L}, 152$.

$55 \mathrm{~L}, 158$.

56 CIr., sobre el empleo de este usquema sintáctico en el español de la ćpoca clásica, Rafael Cano Aguilar "Cambios de construcción verbal en español clásico", en Boletín de la Real Academia Española, 64, 1984, págs. 203-255 (especialmente págs. 248-254) y "Sobre el régimen de las oraciones completivas en español clásico", en Philologica Hispaniensia in Honorem M. Alvar, II, Madrid, 1985, págs. 81-93.

${ }^{57} \mathrm{~L}, 149$.
58 L, 156.
59 L, 147.
60 L, 158.
61 L, 160. 
14.- Intercalación de adverbio entre verbo auxiliar y verbo principal

Son, evidentemente, derivadas de la interferencia de modelos franceses aplicables al orden de palabras en la frase verbal esquemas sintácticos como los siguientes: "Los Gefes de Brigada Pablo Louverture y Carlos Bilite se han particularmente distinguido en esta operación" 63; "Está expresamente mandado a todos los Comandantes Militares..." 64; "A la gran Bahía de Samaná donde se encuentra naturalmente formado el Puertto más vastto y seguro" 65 .

Podrían añadirse sin dificultad otros rasgos, similares a los hasta aquí expuestos, de la documentación que examinamos y relacionables, del mismo modo, con procesos de interferencia del código lingüístico francés $\left(\mathrm{L}_{1}\right.$ sobre el español local de Santo Domingo $\left(L_{2}\right)$. Creo, sin embargo, que son suficientes los datos de índole morfosintáctica, aportados en estas páginas, sumados a los de nivel léxico facilitados en otro trabajo anterior elaborado sobre los mismos materiales ${ }^{66}$ para dar por demostrado el carácter ampliamente interferido de los textos considerados y, sobre esta base empírica, la significativa adecuación de los resultados obtenidos en nuestro análisis respecto a la hipótesis causal que postula la relevancia de los proceso de interferencia de $L_{1}$ sobre $L_{2}$ en lo que toca a la producción de los rasgos caracterízadores de los estadios avanzados de interlenguaje.

Me sentiría ampliamente satisfecho si los datos presentados en este trabajo, además de su mayor o menor interés en el ámbito teórico al que acabo de referirme, pueden ser de alguna utilidad para atraer la atención de los especialistas hacia un ámbito de estudio (el del contacto lingüístico entre el español y el francés / créole haitiano en el área territorial dominicana) que, a mi parecer, debe ser considerado como de especial relevancia no sólo en relación con el planteamiento genérico de los procesos de contacto lingüístico que han tenido lugar en el español de América como conjunto sino, y aún más, en cuanto al análisis específico de los fenómenos de interferencia que se han producido en Hispanoamérica entre el español y el francés los cuales, como con acierto to ha expresado recientemente Philippe Cahuzac ${ }^{67}$, requieren de modo cada vez más urgente una apreciación, pormenorizada y de conjunto, que aún no se les ha dedicado.

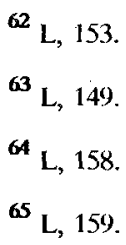

${ }^{66}$ Véase mi trabajo citado en la nota 19. En relación con la importancia de los procesos de interferencia léxica en la producción de interlenguajes véanse C. Pfaff "Constraints on language mixing: intrasentential codeswitching and borrowing in Spanish-English" en Language, 55, 1979, págs. 291-318; J. Schumann The Pidginization Process. A Model for Second Language Acquisition, Rowley, 1978, y C. Facrch y G. Kasper (cds.) Strategies in Interlanguage Communication, Londres-Nueva York, 1983.

67 "Approche méthodologique de l'étude des gallicismes en espagnol d'Amérique", en Hommage a Bernard Pottier, I, Paris, 1988, págs. 127-141. 